\title{
UM EXPERIMENTO SIMPLES PARA MOSTRAR A CONSERVAÇÃO DO MOMENTO LINEAR
}

\author{
VANESSA DA Silva FERREIRA ${ }^{1,2 *}$ \\ Paulo Simeão CARVAlHo $2,3 \dagger$ \\ ${ }^{1}$ Departamento de Física e Astronomia, Instituto de Física dos Materiais da \\ Universidade do Porto, Faculdade de Ciências da Universidade do Porto, \\ Rua do Campo Alegre s/n, Porto, Portugal, CEP 4169-007 \\ ${ }^{2}$ Departamento de Física, Universidade Federal Rural do Rio de Janeiro, \\ Rodovia BR 465, Km 07, s/n - Zona Rural, Seropédica, Brasil, CEP 23890-000 \\ ${ }^{3}$ Unidade de Ensino das Ciências, \\ Rua do Campo Alegre s/n, Porto, Portugal, CEP 4169-007
}

\begin{abstract}
Resumo
A lei de conservação do momento linear de um sistema é, normalmente, ensinada nas aulas de Física em contextos de choques e colisões. Com muita frequência, os alunos ficam convencidos que essa lei apenas se aplica a tais situações, o que constitui uma limitação na aprendizagem e utilização da lei em situações mais gerais. Neste trabalho usamos uma mola elástica para mostrar como esta lei pode ser ensinada num contexto diferente, com muita simplicidade e de forma intuitiva, associando esta lei ao movimento do Centro de Massa (CM) de um sistema.
\end{abstract}

Palavras-chave: Momento Linear; Lei de Conservação do Momento Linear; Centro de Massa.

\footnotetext{
*E-mail: tr.vanessa.ferreira@gmail.com

${ }^{\dagger}$ E-mail:psimeao@fc.up.pt
} 


\begin{abstract}
The linear momentum conservation law is usually taught in physics classes in contexts of collisions. Too often, students are convinced that this law only applies to those situations, which constitutes a limitation in the learning process and use of this law in more general situations. In this work, we use an elastic spring to show how this law can be taught in a different context, in a very simply and intuitive way, associating this law with the motion of the Center of Mass (CM) of a system.
\end{abstract}

Keywords: Linear Momentum; Momentum Conservation Law; Center of Mass.

\title{
1 Introdução
}

A lei da conservação do momento linear diz-nos que se a resultante das forças externas que atuam sobre um sistema for nula, então o momento linear desse sistema é conservado. Esta lei tem uma implicação imediata sobre o movimento do Centro de Massa (CM) do sistema: sendo este o representante do movimento coletivo do sistema, como se toda a massa deste estivesse lá localizada, então o momento linear do CM, $\vec{P}_{C M}$, mantém-se inalterado até que uma força externa atue sobre o sistema.

Se o sistema estiver inicialmente em repouso e a resultante das forças externas que atuam sobre ele for nula, então tem-se sempre,

$$
\vec{P}_{C M}=0,
$$

mesmo que, localmente, haja partes do sistema em movimento.

Por tradição, os contextos de choques e colisões são preferencialmente usados nas aulas de Física para ensinar a lei da conservação do momento linear (Serway \& Beichner, 2000). Todavia, esta limitação de contextos faz os alunos acreditar que este conteúdo é específico e só se aplica aos choques e colisões de objetos. Nada mais errado! Quando confrontados com fenómenos em outros contextos (por exemplo, o movimento de objetos no interior de naves espaciais), revelam dificuldade em invocar esta lei. Há, assim, necessidade de variar e diversificar os contextos em que esta lei se aplica.

\section{MONTAGEM EXPERIMENTAL}

Para abordar a lei da conservação do momento linear de um sistema num contexto fora do habitual, usamos uma mola elástica do tipo Slinky sobre uma mesa horizontal. A mola, cujo comprimento natural é de cerca de $5,9 \mathrm{~cm}$, foi alongada até $20,55 \mathrm{~cm}$ como mostra a Figura 1 . Nas extremidades da mola foram colocados dois marcadores coloridos para melhor identificar o movimento dessas extremidades. O Centro de Massa da mola encontra-se localizado a meio, tal como é indicado na figura por uma marca (virtual) a verde. 


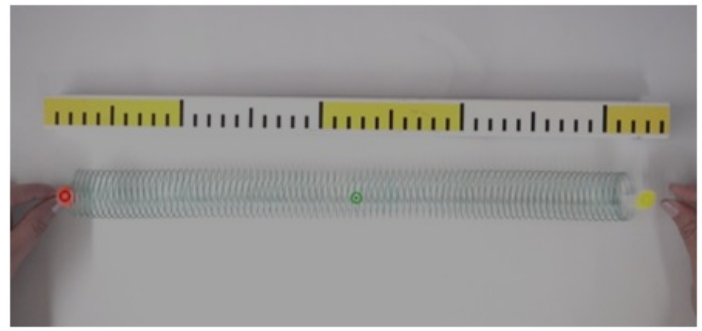

Figura 1: Mola slinky alongada. As extremidades foram identificadas com marcadores coloridos (vermelho e amarelo). O CM da mola aparece identificado (virtualmente) a verde.

Seguidamente, a mola foi largada simultaneamente das duas extremidades. O movimento da mola foi então gravado com uma câmara fotográfica SONY Cybershot DSC-RX100 V, a uma taxa de 250 quadros por segundo. Os dados recolhidos em vídeo foram analisados pelo software Tracker (BROWN \& COX, 2009) e os gráficos criados em MSExcel.

\section{RESULTADOS E DISCUSSÃO}

A Figura 2 mostra o gráfico do movimento das extremidades da mola e do CM, obtidas a partir do vídeo (CARVALHO, 2017) e da análise com o Tracker. O deslocamento simétrico das extremidades permite concluir que estas estão sujeitas a forças simétricas. Contudo, o valor destas forças não é constante.

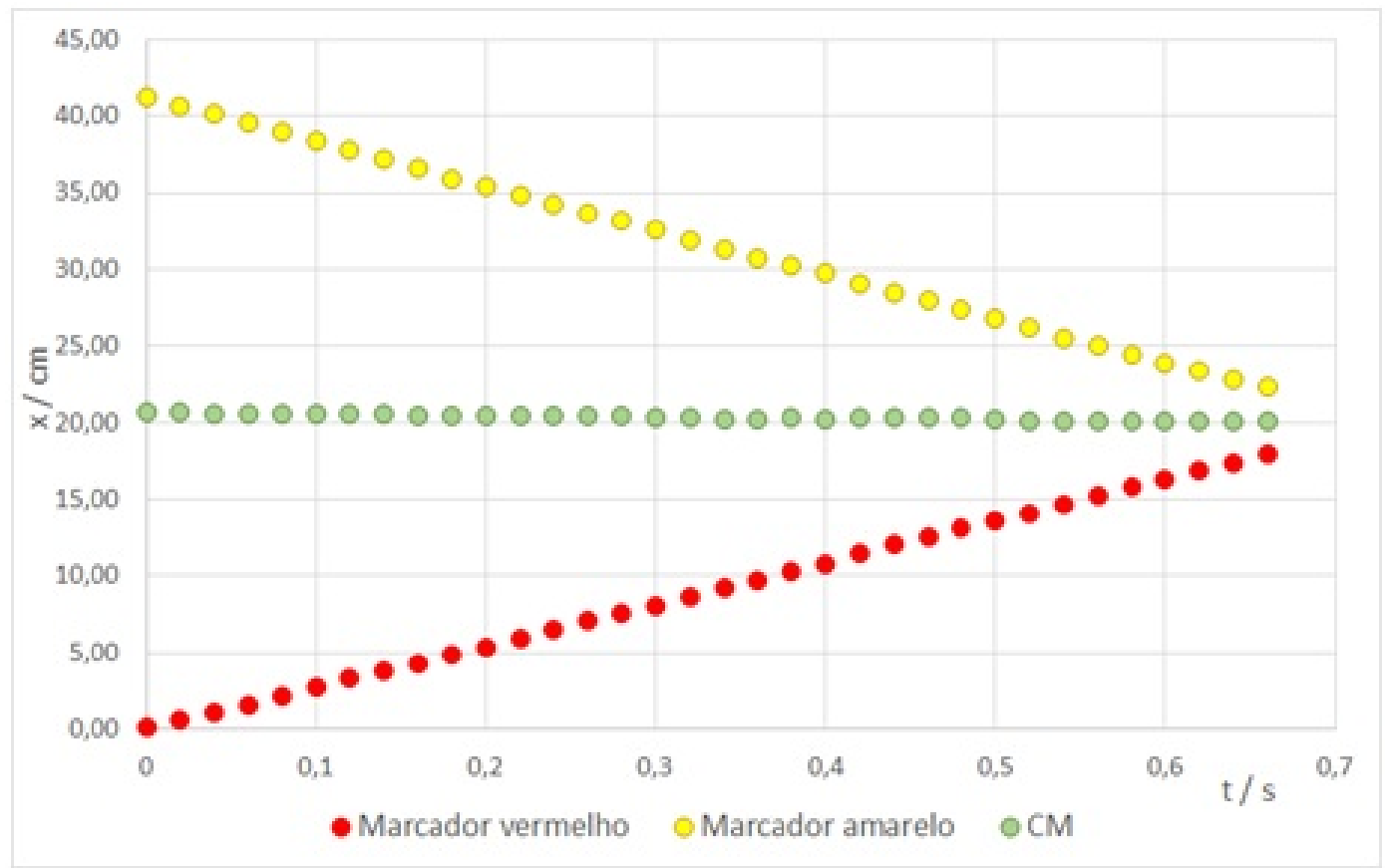

Figura 2: Posição das extremidades vermelha (pontos a vermelho) e amarela (pontos a amarelo), e do CM (pontos a verde) da mola, em função do tempo. 
Uma análise cuidadosa ao movimento da extremidade vermelha (Figura 3) mostra que nos primeiros $0,12 s$, esta extremidade adquire um movimento que pode ser ajustado por uma equação de segundo grau, a qual tipicamente caracteriza um movimento com aceleração constante. $O$ termo quadrático da equação de ajuste representa o dobro do valor da aceleração média na direção do movimento, que neste caso é de aproximadamente $12,5 \mathrm{~cm} / \mathrm{s}^{2}$.

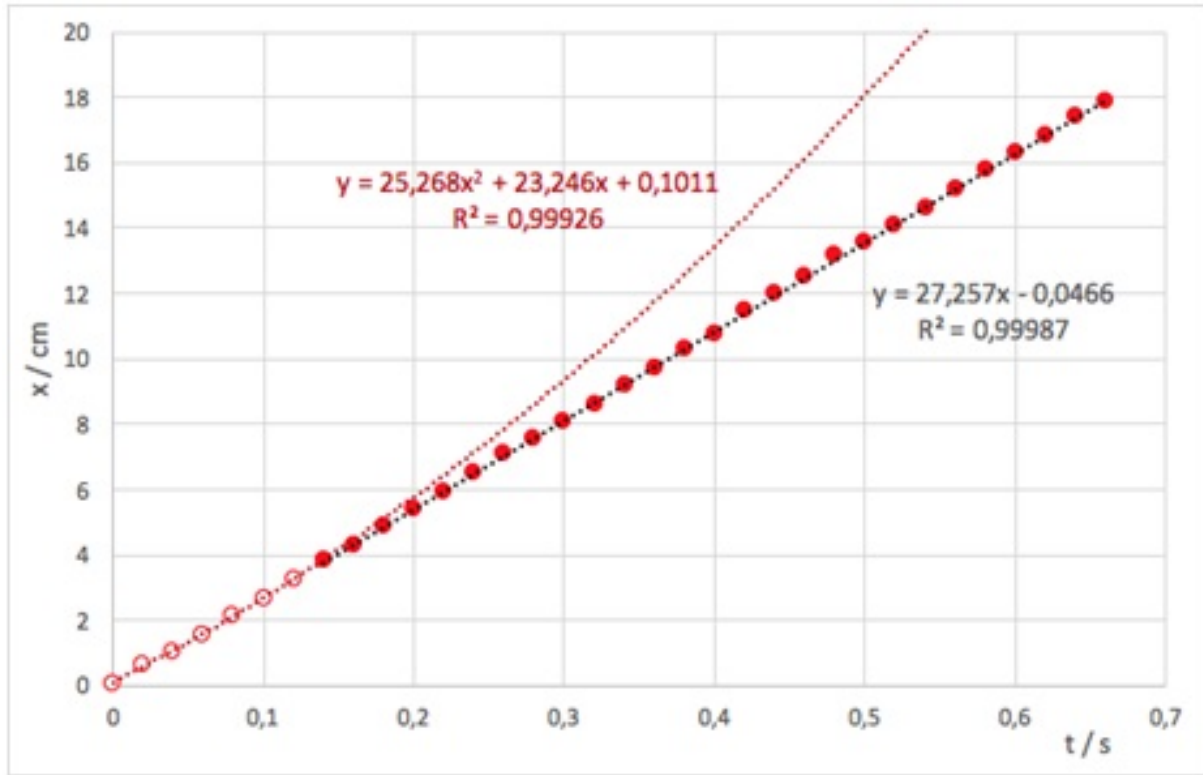

Figura 3: Posição da extremidade vermelha da mola em função do tempo. Nos primeiros $0,12 \mathrm{~s}$, a extremidade possui um movimento acelerado, com uma aceleração média de aproximadamente $12,5 \mathrm{~cm} / \mathrm{s}^{2}$ (linha de ajuste a vermelho ponteado). Após este instante, a extremidade parece apresentar um movimento uniforme (linha de ajuste a preto ponteado). Os valores de $R^{2}$ garantem a qualidade dos ajustes. Não foi feita truncagem aos parâmetros de ajuste..

Após este instante, a extremidade adquire um movimento praticamente uniforme, ajustável por uma relação linear no tempo. Como durante todo o movimento há sempre uma força elástica a atuar sobre a extremidade, este comportamento sugere a existência de uma força de atrito exercida pela superfície sobre a mola, que contraria e equilibra a força elástica.

Um desempenho em tudo semelhante é também observado na extremidade amarela, em que agora a força de atrito exercida pela superfície sobre a mola terá sentido oposto ao descrito anteriormente.

Como o movimento das extremidades é simétrico, a posição do CM da mola permanece inalterada durante todo o movimento. Ou seja, confirma-se que $\vec{P}_{C M}=\overrightarrow{0}$.

Assim, o sistema apresentado (a mola) contém elementos que estão em movimento, mas como as forças externas se equilibram e as forças internas não contribuem para o movimento do centro de massa do sistema, o momento linear do CM não sofre qualquer alteração. Este exemplo constitui um contexto diferente, embora enriquecido e inovador, para o ensino da lei da conservação do momento linear numa situação fora do contexto habitual das colisões, permitindo assim mostrar que esta lei pode ser abordada com grande generalidade. 


\section{Conclusão}

$\mathrm{O}$ experimento aqui apresentado sugere uma abordagem didática simples e muito intuitiva para o ensino da lei da conservação do momento linear. Ele permite explorar esta lei num contexto em que a resultante das forças externas que atuam sobre o sistema é nula, situação diferente das habituais colisões e dos choques, e também associar com facilidade a lei da conservação do momento linear ao comportamento do CM do sistema.

Este trabalho deve ser encarado pelo professor de Física, como uma sugestão para a utilização dessa lei para interpretar situações físicas mais gerais, e assim evitar limitar a aprendizagem dos alunos a contextos demasiado académicos e particulares.

A atividade experimental é de simples implementação em qualquer escola e envolve recursos materiais de baixo custo. A sua realização constitui uma primeira análise conceptual e qualitativa do fenômeno. A filmagem do movimento da mola e consequente estudo, deve ser usada de forma complementar para se obter uma abordagem quantitativa do fenômeno.

\section{Agradecimentos}

Vanessa Ferreira agradece ao Programa de Educação Tutorial - PET, do Ministério da Educação do Brasil, por ter proporcionado o estágio na Universidade do Porto. Este trabalho foi financiado pela Fundação para a Ciência e a Tecnologia, Projeto UID/NAN/50024/2013.

\section{Referências}

[1] BROWN, D., COX, A.J.,Innovative Uses of Video Analysis, The Physics Teacher 47, 145150 (2009).

[2] CARVALHO, P.S., Mola Slinky em movimento horizontal a 250fps. Disponível em: https://youtu.be/om0ND-wYe1U , 2017. Acesso: 22/10/2017.

[3] SERWAY, R.A, BEICHNER, R.J., Physics for Scientists and Engineers with Modern Physics, 5th Ed., Orlando, Brooks/Cole, 2000. pp. 251-281. 